\title{
A familial case of limb-girdle muscular dystrophy with CAV3 mutation
}

\author{
Seungbok Lee ${ }^{1 \oplus}$, Sesong Jang ${ }^{2 \oplus}$, Youngkyu Shim ${ }^{\oplus}$, Woo Joong Kim ${ }^{1 \oplus}$, Soo Yeon Kim ${ }^{1 \oplus}$, Anna Cho ${ }^{3 \oplus}$, Hunmin $\mathrm{Kim}^{4 \oplus}$, \\ Jong-Il Kim ${ }^{2}$, Byung Chan $\mathrm{Lim}^{1 \oplus}$, Hee Hwang ${ }^{4}{ }^{\oplus}$, Jieun $\mathrm{Choi}^{5 \oplus}$, Ki Joong Kim ${ }^{1 \oplus}$, and Jong Hee Chae ${ }^{1 \oplus}$ \\ ${ }^{1}$ Department of Pediatrics, Seoul National University Children's Hospital, Seoul National University College of Medicine, Seoul, Korea \\ ${ }^{2}$ Genomic Medicine Institute (GMI), Medical Research Center, Seoul National University, Seoul, Korea \\ ${ }^{3}$ Department of Pediatrics, Ewha Womans University School of Medicine, Seoul, Korea \\ ${ }^{4}$ Department of Pediatrics, Seoul National University Bundang Hospital, Seongnam, Korea \\ ${ }^{5}$ Department of Pediatrics, SMG-SNU Boramae Medical Center, Seoul, Korea
}

Limb-girdle muscular dystrophy (LGMD) is a group of muscular dystrophies that has extremely heterogeneous clinical features and genetic background. The caveolin-3 gene (CAV3) is one of the causative genes. LGMD appears as a clinical continuum, from isolated skeletal muscle involvement to long QT syndrome. Here we report two patients without apparent muscle weakness in a family with CAV3 mutation.

A 7-month-old Korean boy visited our muscle clinic because of an incidental finding of elevated serum creatine kinase (CK) concentration (680 IU/L, reference range, 20-270 IU/L) without clinical symptoms. The patient was born after an uneventful pregnancy and showed normal developmental milestones. He developed pseudohypertrophy of his calf muscle during the follow-up. We obtained a muscle biopsy at age 14 months, which showed size variations and degenerating/regenerating myofibers with endomysial fibrosis and immunohistochemical evidence of normal dystrophin. Under the impression of LGMD, we performed target panel sequencing and identified a heterozygous in-frame mutation of CAV3, c.307_312delGTGGTG (p.Val103_Val104del). Immunohistochemical staining of muscle indicated complete loss of caveolin-3 compared with normal control muscle, which supported the variant's pathogenicity. We performed segregation analysis and found that the patient's mother had the same variant with elevated serum CK level (972 IU/L).

We report on autosomal dominant familial caveolinopathy caused by a pathogenic variant in CAV3, which was asymptomatic until the fourth decade. This case highlights the utility of next generation sequencing in the diagnosis of muscular dystrophies and the additive role of muscle biopsy to confirm the variants.

Key words: Muscular dystrophies, limb-girdle, Caveolin 3, High-throughput nucleotide sequencing, Creatine kinase.

\section{Introduction}

Limb-girdle muscular dystrophy (LGMD) is a group of mus- cular dystrophies that show extremely heterogeneous clinical features and genetic backgrounds. Patients with LGMD commonly show elevated creatinine kinase (CK) level and progressive

\footnotetext{
Received: 8 August 2019, Revised: 2 September 2019, Accepted: 11 September 2019, Published: 31 December 2019

*Corresponding author: Jong Hee Chae, M.D., Ph.D. (iD https://orcid.org/0000-0002-9162-0138

Department of Pediatrics, Seoul National University Children's Hospital, Seoul National University College of Medicine, 101 Daehak-ro, Jongno-gu, Seoul 03080, Korea.

Tel: +82-2-2072-3622, Fax: +82-2-743-3455, E-mail: chaeped1@snu.ac.kr

Conflict of interest: The authors declare that they do not have any conflicts of interest.

(c) This is an open-access article distributed under the terms of the Creative Commons Attribution Non-Commercial License (http://creativecommons.org/licenses/by-nc/4.0/) which permits unrestricted non-commercial use, distribution, and reproduction in any medium, provided the original work is properly cited.

(c) Copyright 2019 by the Korean Society of Medical Genetics and Genomics 
weakness of the proximal muscles, which usually starts after 2 years of age. However, the variety of causal genes or genetic loci associated with this disease suggests that the clinical features can vary widely between patients seen in clinical practice and the differential diagnosis is a challenge [1].

According to the concept of causal genes and the manner of inheritance, LGMD is now categorized into eight autosomal dominant (LGMD1A-1H) and 26 autosomal recessive (LGMD2A$2 A)$ subgroups [2]. However, it can be difficult to differentiate subtypes for several reasons: 1) the large number of subgroups showing a broad spectrum of clinical manifestation, 2) inheritance of genes in both autosomal dominant and recessive manners, and 3) exhibition of the LGMD phenotype by several other muscular dystrophies that are not yet classified as LGMD. Therefore, comprehensive molecular diagnosis in addition to the classic pathological approaches is needed to identify this disease group. High-throughput technologies such as next generation sequencing (NGS) can be helpful in the genetic diagnosis of inherited myopathies because a wide variety of genes needs to be targeted [3].

Here we report on two cases of caveolinopathy without apparent muscle weakness that were confirmed by target gene panel sequencing and analysis of muscle pathology.

\section{Case}

A previously healthy 7-month-old Korean boy visited the neuromuscular clinic of Seoul National University Children's Hospital because of an incidental finding of elevated CK concentration (680 IU/L, reference range, 20-270 IU/L). Neither clinical symptoms nor abnormalities on neurological examination were found at his first visit. During the follow-up period, increased serum CK concentration (hyperCKemia) persisted (760-2,140 IU/ L) and pseudohypertrophy developed in his calf muscle. He was admitted for a muscle biopsy at the age of 14 months. At that time, he could run and stand up by himself, and showed normal motor development. Muscle power was grade $\mathrm{V}$ in all extremities, and deep tendon reflexes were intact and symmetric for the knee jerk and ankle jerk reflexes. The muscle biopsy showed moderate size variations of myofibers, with some degenerating and regenerating fibers and endomysial/perimysial fibrosis with mild fatty change, which are consistent with muscular dystrophy. Immunohistochemical staining for dystrophin 1, 2, and 3 showed weak activity compared with normal control tissue.

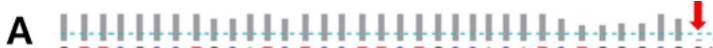

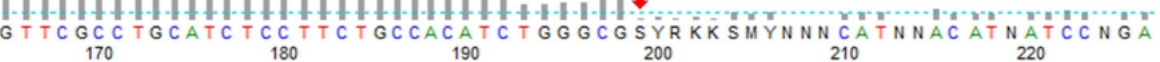

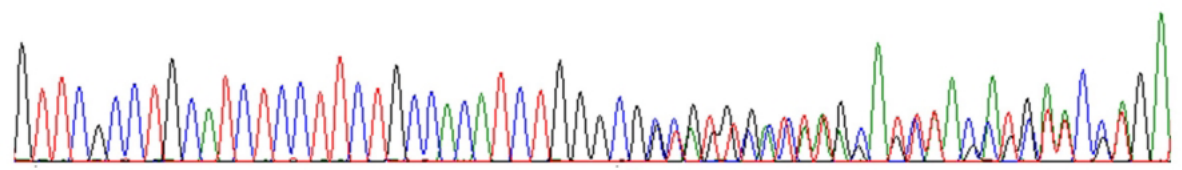

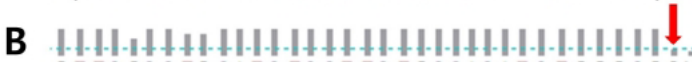
GTTCGCCTGCATCTCCT TCTGCCACATC TGGGCGSYRK KS MNN GNAKNNAC AN NANCNWGATC

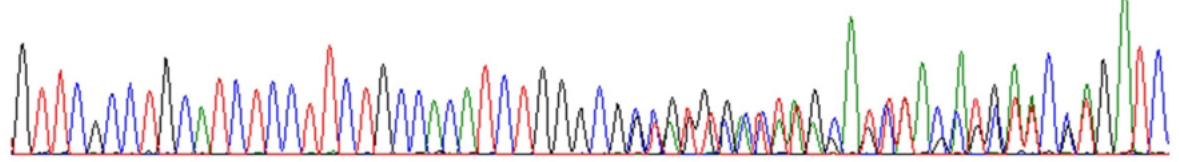

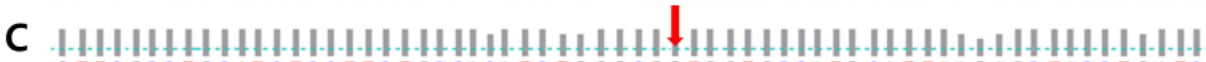

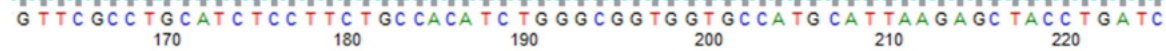
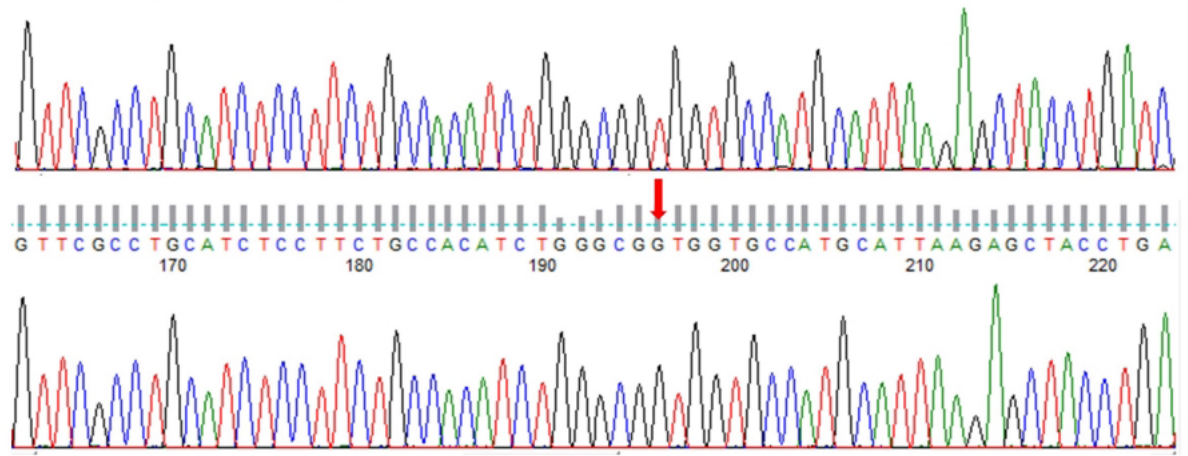

Fig. 1. Electropherogram of Sanger sequencing. The patient $(A)$ and his mother (B) share the same heterozygous mutation on CAV3, while his father (C) and sister (D) do not have the mutation. The red arrows indicate the genomic position where the deletion starts. 
Genetic testing for $D M D$, using multiplex ligation-dependent probe amplification and Sanger sequencing of the whole gene, did not find any pathogenic variants.

At 3 years of age, the patient exhibited ankle contracture on both sides, which resulted in limited dorsiflexion. He was referred to the rehabilitation medicine clinic, which recommended home physical therapies including stretching and passive range of motion exercises. He continued to visit the hospital for a regular check-up every 6 months and showed normal development and tolerable exercise performance until the last visit at the age of 16 years. Regular echocardiography and electrocardiography indicated normal heart function.

He underwent target gene panel sequencing for molecular diagnosis, which revealed a heterozygous in-frame deletion of CAV3 c.307_312delGTGGTG (p.Val103_Val104del) (Fig. 1). Segregation analysis was performed for all family members and this analysis showed that the patient's mother had the same heterozygous variant. She was asymptomatic and had no abnormalities on neurological examination but exhibited hyperCKemia (972 IU/L). For the functional validation of this variant, we performed immunohistochemical staining of frozen muscle specimen using antibody to caveolin, which revealed a definitively low caveolin-3 expression compared with normal control tissue (Fig. 2). This finding supported the evidence that the deletion was a pathogenic variant.

\section{Discussion}

Here we report on autosomal dominant familial caveolinopathy caused by a pathogenic variant in CAV3. Based on confirmation of the CAV3 mutation using NGS, the boy and his mother were diagnosed with caveolinopathy, each presented as LGMD1C and isolated hyperCKemia, respectively. They did not show any muscle weakness until the last follow-up, when the patient was 16 years old and his mother in the fourth decade, only exhibiting elevated CK levels. However, since the proband showed pseudohypertrophy, mild ankle contracture, and pathologic features of muscular dystrophy in muscle biopsy, he could be diagnosed as LGMD1C. In addition, the immunohistochemical staining for caveolin-3 confirmed that the mutation contributed to the pathogenesis.

CAV3 encodes caveolin-3 protein, which is the muscle-specific form of the caveolin protein family. Caveolins are the principal components of caveolae, which are subcompartments of the plasma membrane [4]. Caveolin-3 plays a critical role in muscle development and physiology, such as the transmission of the contractile impulse and stability of the muscle cell membrane as a component of the dystrophin-glycoprotein complex [5].

The phenotype of caveolinopathy is extremely heterogeneous and includes rippling muscle disease, LGMD, long QT syndrome, distal myopathy, exercise intolerance, and isolated hyperCKemia [5]. Its initial presentation varies from myotonia or progressive weakness to sudden infant death. The onset age is also extremely variable and ranges from early childhood to the mid-60s, for
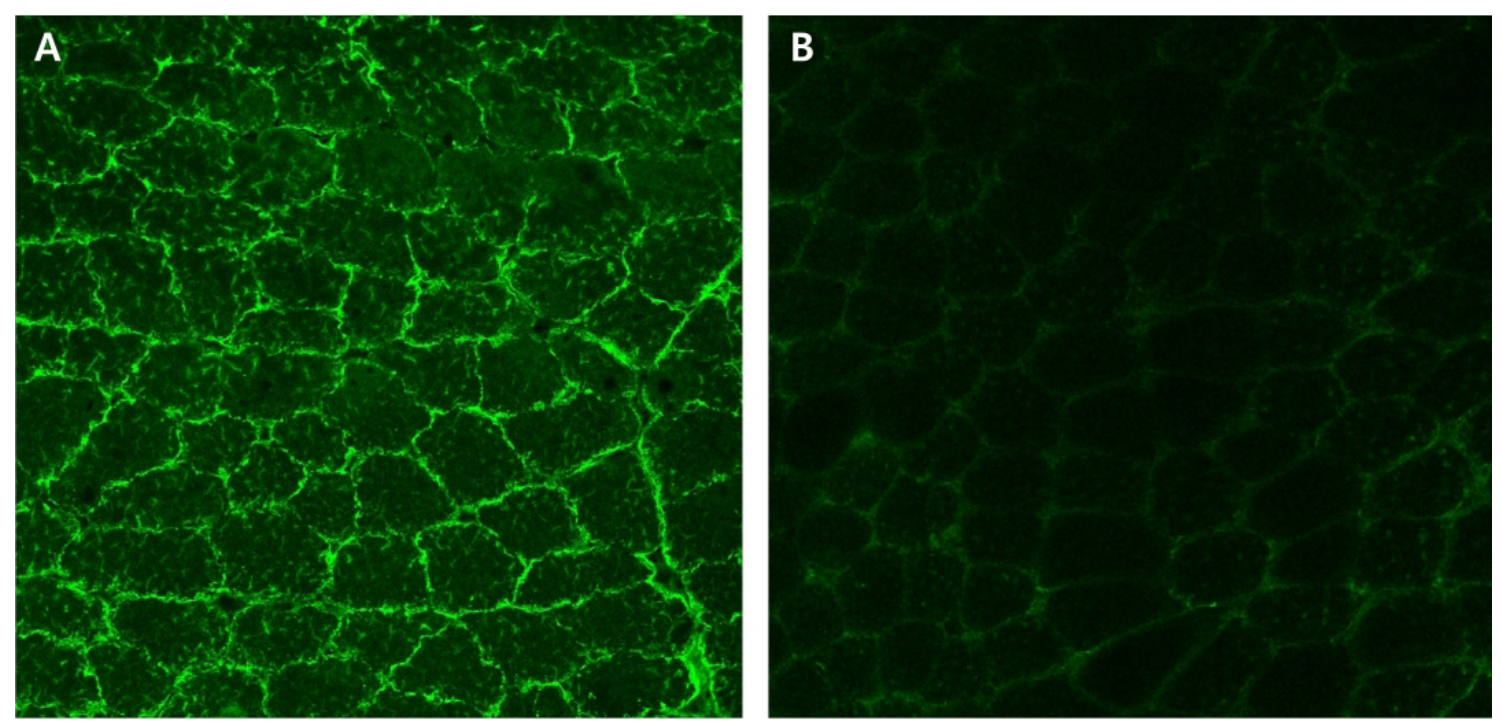

Fig. 2. Immunofluorescence staining of caveolin-3 protein. The signal intensity is much lower in our patient muscle biopsy sample (B) compared to that of normal control $(A)$. 
both CAV3-related LGMD and CAV3-related myotonia [6-8]. In addition, intrafamilial phenotypic variability has been reported in previous studies [5,9]. Our cases also showed an example of intrafamilial variability; the symptoms of pseudohypertrophy and ankle contracture are only observed in the index case. In addition, although there are a couple of reports of patients with isolated hyperCKemia only [10] and both of our cases have no exercise intolerance to date, considering its phenotypic variability, the patients may need regular follow-up to check for lateonset symptoms.

According to the Human Gene Mutation Database, 41 mutations in CAV 3 have been reported to date, and three of these are small deletions [11]. One of these mutations is the same as that found in our patient, and this mutation has also been reported in a Korean patient with rippling muscle disease [12]. This finding suggests the ethnic specificity of this CAV3 mutation (p.Val103_ Val104del). Considering the variable clinical manifestations, we may assume that there are asymptomatic or minimally affected carriers of the mutation in a Korean population.

To date, the phenotype-genotype correlation remains unclear. Patients with variants in the same genomic position have been shown to have various clinical features [5,9]. The in-frame deletion in our patients might be associated with the mild phenotype, such as isolated hyperCKemia. However, another in-frame deletion has been reported in a large family with interfamilial variability, including early adult-onset LGMD. This finding suggests that modifiers such as rare single nucleotide polymorphisms or other epigenetic determinants may regulate the CAV3-related pathways and contribute to the clinical features. Further research is needed to confirm the genotype-phenotype correlation in caveolinopathy.

LGMD is a very heterogeneous disorder caused by a variety of genes, and advance in genetic diagnosis have increased the diagnostic rates of LGMD [3]. However, genomic variants are often hard to interpret in many cases, and segregation or functional validations are essential for making the final molecular diagnosis. Muscle pathology is one of the easiest ways to prove pathogenicity. We were able to show the complete loss of caveolin-3 using immunohistochemical staining which, to our knowledge, is the first demonstration of the variant. As a result of our analysis, the patient was finally diagnosed using NGS after 15 years of follow-up. This type of genetic diagnosis allows for the prediction of disease prognosis and provides a proper guide for patients that includes, for example, recommendations for cardiology consultation. Recently, the clinical utility of NGS, particularly for neuromuscular disease, has been shown clearly, but traditional diagnostic approaches such as the muscle biopsy should be considered because they may also provide important clues for the correct diagnosis.

\section{Acknowledgements}

Jong Hee Chae was supported by a Korea Health Technology R\&D Project grant through the Korea Health Industry Development Institute, funded by the Ministry of Health and Welfare, Republic of Korea (grant number: HI13C-1468).

\section{References}

1. Liewluck T, Milone M. Untangling the complexity of limb-girdle muscular dystrophies. Muscle Nerve 2018;58:167-77.

2. OMIM-Online Mendelian Inheritance in Man ${ }^{\circledR}$. [https://www.omim.org/]

3. Özyilmaz B, Kirbiyik Ö, Özdemir TR, Kaya Özer Ö, Kutbay YB, Erdogan KM, et al. Impact of next-generation sequencing panels in the evaluation of limb-girdle muscular dystrophies. Ann Hum Genet 2019;83:331-47.

4. Minetti C, Sotgia F, Bruno C, Scartezzini P, Broda P, Bado M, et al. Mutations in the caveolin-3 gene cause autosomal dominant limb-girdle muscular dystrophy. Nat Genet 1998;18:365-8.

5. Gazzerro E, Sotgia F, Bruno C, Lisanti MP, Minetti C. Caveolinopathies: from the biology of caveolin-3 to human diseases. Eur J Hum Genet 2010;18:137-45.

6. Aboumousa A, Hoogendijk J, Charlton R, Barresi R, Herrmann R, Voit $T$, et al. Caveolinopathy--new mutations and additional symptoms. Neuromuscul Disord 2008;18:572-8.

7. Fulizio L, Nascimbeni AC, Fanin M, Piluso G, Politano L, Nigro $V$, et al. Molecular and muscle pathology in a series of caveolinopathy patients. Hum Mutat 2005;25:82-9.

8. Papadopoulos C, Papadimas GK, Kekou K, Spengos K, Svigou M, KitsiouTzeli S, et al. Caveolinopathies in Greece. Neurologist 2015;20:8-12.

9. Fischer $D_{1}$ Schroers $A_{1}$ Blümcke I, Urbach $H_{1}$ Zerres $K_{1}$ Mortier $W_{1}$ et al Consequences of a novel caveolin-3 mutation in a large German family. Ann Neurol 2003;53:233-41.

10. Reijneveld JC, Ginjaar IB, Frankhuizen WS, Notermans NC. CAV3 gene mutation analysis in patients with idiopathic hyper-CK-emia. Muscle Nerve 2006;34:656-8.

11. Stenson PD, Mort M, Ball EV, Evans K, Hayden M, Heywood S, et al. The Human Gene Mutation Database: towards a comprehensive repository of inherited mutation data for medical research, genetic diagnosis and next-generation sequencing studies. Hum Genet 2017;136:665-77.

12. Bae JS, Ki CS, Kim JW, Suh YL, Park MS, Kim BJ, et al. A novel in-frame deletion in the CAV3 gene in a Korean patient with rippling muscle disease. J Neurol Sci 2007;260:275-8. 